Geometry $\&$ Topology

Volume 9 (2005) 95-119

Published: 22 December 2004

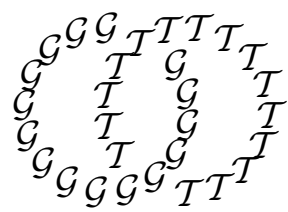

\title{
Distances of Heegaard splittings
}

\author{
Aaron ABRAMS \\ SAUL SCHLEIMER \\ Department of Mathematics, Emory University \\ Atlanta, Georgia 30322, USA \\ and \\ Department of Mathematics, Rutgers University \\ Piscataway, New Jersey 08854, USA \\ Email: abrams@mathcs.emory.edu and saulsch@math.rutgers.edu \\ URL's: http://www. mathcs.emory.edu/ abrams \\ http://www. math.rutgers.edu/ $\sim_{\text {saulsch }}$
}

\begin{abstract}
J Hempel [Topology, 2001] showed that the set of distances of the Heegaard splittings $\left(S, \mathcal{V}, h^{n}(\mathcal{V})\right)$ is unbounded, as long as the stable and unstable laminations of $h$ avoid the closure of $\mathcal{V} \subset \mathcal{P} \mathcal{M L}(S)$. Here $h$ is a pseudo-Anosov homeomorphism of a surface $S$ while $\mathcal{V}$ is the set of isotopy classes of simple closed curves in $S$ bounding essential disks in a fixed handlebody.

With the same hypothesis we show the distance of the splitting $\left(S, \mathcal{V}, h^{n}(\mathcal{V})\right)$ grows linearly with $n$, answering a question of A Casson. In addition we prove the converse of Hempel's theorem. Our method is to study the action of $h$ on the curve complex associated to $S$. We rely heavily on the result, due to H Masur and Y Minsky [Invent. Math. 1999], that the curve complex is Gromov hyperbolic.
\end{abstract}

AMS Classification numbers Primary: 57M99

Secondary: $51 \mathrm{~F} 99$

Keywords: Curve complex, Gromov hyperbolicity, Heegaard splitting

Proposed: Martin Bridson

Received: 5 June 2003

Seconded: Cameron Gordon, Joan Birman

Revised: 20 December 2004

(C) Geometry $8 \mathcal{T}$ Topology Publications 


\section{Introduction}

J Hempel [8] introduced a new measure of the complexity of a Heegaard splitting called the distance of the splitting. This definition is a conscious extension of A Casson and C Gordon's notion of strong irreducibility [3]. Indeed, a Heegaard splitting is

- reducible if and only if its distance is 0 ,

- weakly reducible if and only if its distance is at most 1 , and

- strongly irreducible if and only if its distance is at least 2 .

Hempel's distance derives its name from the curve complex; the distance of a splitting is exactly the distance between the two handlebodies, thought of as subsets of the curve complex associated to the splitting surface. In particular the distance does not rely on any particular diagram for the splitting.

Casson and Gordon produce examples of strongly irreducible splittings by taking an existing splitting of $S^{3}$ and altering the gluing map by high powers of a certain Dehn twist. It is clear that the Dehn twist must be carefully chosen; a Dehn twist about a curve which bounds a disk in one of the two handlebodies leaves the splitting unchanged.

Hempel obtains examples of high distance splittings using a construction due to T Kobayashi 11. Instead of $S^{3}$ he begins with the double of a handlebody $V$. Instead of a Dehn twist he iterates a certain pseudo-Anosov map $h$ on $S=$ $\partial V$. Analyzing the dynamics of $h$ acting on the space $\mathcal{P} \mathcal{M L}(S)$ of projective measured laminations reveals that the set of distances, obtained by altering the original gluing by $h^{n}$, is unbounded. We sketch Hempel's proof in Section 2 , Again, one must be careful when choosing $h$; if $h$ extends over the handlebody then the splitting remains unchanged.

We remark that the second author has proved that each fixed 3-manifold has a bound on the distances of its Heegaard splittings. Thus the splittings provided by Hempel's theorem must represent infinitely many different 3-manifolds.

This paper is part of an ongoing program to understand handlebodies and Heegaard splittings from the point of view of the curve complex. The fundamental ingredient underlying our approach is the result of H Masur and Y Minsky 14] that the curve complex is $\delta$-hyperbolic. This allows us to study the dynamics of $h$ acting on the curve complex. We are thus able to both strengthen Hempel's theorem and to prove a converse. 
Theorem 1.1 Fix a handlebody $V$ with genus at least two and set $S=\partial V$. Fix also a pseudo-Anosov map $h: S \rightarrow S$. Let $\mathcal{V}$ be the set of isotopy classes of simple closed curves in $S$ which bound disks in $V$. Let $\alpha(h)$ denote the average displacement of $h$. The following are equivalent:

K: The stable and unstable laminations of $h$ are each of full type with respect to some pants decompositions of $V$.

M: In $\mathcal{P} \mathcal{M L}(S)$, the stable and unstable laminations of $h$ are contained in the Masur domain of $V$.

$\mathbf{H}$ : In $\mathcal{P} \mathcal{M L}(S)$, the stable and unstable laminations of $h$ are not contained in the closure of $\mathcal{V}$.

B: In the curve complex, the projection of $\mathcal{V}$ onto an invariant axis for $h$ has finite diameter.

Lin: There is a constant $K>0$ so that for any $n \in \mathbb{N}$ the distance of the Heegaard splitting $\left(S, \mathcal{V}, h^{n}(\mathcal{V})\right)$ lies between $n \cdot \alpha(h)-K$ and $n \cdot \alpha(h)+K$.

Unb: The set of distances of $\left\{\left(S, \mathcal{V}, h^{n}(\mathcal{V})\right) \mid n \in \mathbb{N}\right\}$ is unbounded.

The terms average displacement, full type, Masur domain, and invariant axis are defined in Definitions 3.1, 11.2 (or the paper [1]), 10.1] (or the paper [12]), and 5.6 respectively.

Hempel 8 proved that $\mathbf{H}$ implies Unb. Section 2 gives Hempel's definition of distance as well as a sketch of his proof.

In this paper we introduce the condition $\mathbf{B}$ and prove in Section 8 its equivalence with $\mathbf{H}$. This strengthens Hempel's theorem as $\mathbf{B}$ (and hence $\mathbf{H}$ ) implies Lin. (Note that Lin implies Unb by the fact, also contained in 14, that $\alpha(h)>0$.) To prove this we first develop several tools from $\delta$-hyperbolic geometry in Sections 3 through 6. These arguments are written out carefully to emphasize their synthetic nature, in particular the fact that they apply to spaces (such as the curve complex) which are not locally compact. In Section 17, we apply these tools to the curve complex, as allowed by Masur and Minsky's theorem, to obtain Corollary 17.5, B implies Lin. The argument in Section 8 shows that B is an accurate translation of $\mathbf{H}$ to the geometric language of the curve complex. This relies on E Klarreich's characterization [10] of the Gromov boundary of the complex of curves as the space of unmeasured, minimal laminations.

In order to prove the converse, namely that Unb implies $\mathbf{B}$, we use the more recent theorem of Masur and Minsky [13] that handlebody sets are quasi-convex 
subsets of the curve complex. This is carried out in Section 9. The equivalence of $\mathbf{H}, \mathbf{M}$, and $\mathbf{K}$ is established in the final two sections.

Acknowledgements We thank Howard Masur for many interesting conversations and for showing us the proof of Lemma 11.7. AA was supported in part by NSF grant DMS-0089927; SS was supported in part by NSF grant DMS-0102069.

\section{Hempel's argument}

Beginning with a few definitions, this section states Hempel's theorem and sketches a proof.

\subsection{Terminology for Heegaard splittings}

A handlebody is a compact three-manifold which is homeomorphic to a closed regular neighborhood of a finite, polygonal, connected graph embedded in $\mathbb{R}^{3}$. The genus of the handlebody is the genus of its boundary. A properly embedded disk $D$ in a handlebody $V$ is essential if $\partial D$ is not null-homotopic in $\partial V$.

Fix handlebodies $V$ and $W$ of the same genus. Let $S=\partial V$. Glue $V$ and $W$ together via a homeomorphism $f: \partial V \rightarrow \partial W$. We will consistently use $\mathcal{V}$ to denote the handlebody set: the set of (isotopy classes of) curves in $S$ which bound essential disks in $V$. Let $\mathcal{W}$ denote the set of curves in $S$ which, after gluing, bound essential disks in $W$. Then the data $(S, \mathcal{V}, \mathcal{W})$ give a Heegaard splitting. Note that a Heegaard splitting specifies a closed orientable threemanifold. The surface $S$ is referred to, in other literature, as the Heegaard splitting surface.

Definition 2.1 The distance of the splitting $(S, \mathcal{V}, \mathcal{W})$ (see $[8]$ ) is the smallest $n \in \mathbb{N}$ such that there are $n+1$ essential simple closed curves $\alpha_{i} \subset S$ with the following properties:

- $\alpha_{0} \in \mathcal{V}$ and $\alpha_{n} \in \mathcal{W}$ and

- $\alpha_{i} \cap \alpha_{i+1}=\emptyset$ for $i=0, \ldots, n-1$.

Now suppose that $h$ is a homeomorphism of $S=\partial V$. Then the set of curves $h(\mathcal{V})$ also defines a handlebody and $(S, \mathcal{V}, h(\mathcal{V}))$ also specifies a Heegaard splitting. 


\subsection{Hempel's theorem}

As above fix a handlebody $V$ of genus at least two and set $S=\partial V$. We will freely use known facts about $\mathcal{P} \mathcal{M L}(S)$, the projectivization of the space of measured laminations, and about the mapping class group of $S$. (See [5] or [9] for extensive discussion of these objects.)

For convenience of notation we occasionally blur the distinction between an object and its isotopy class. That said, let $\mathcal{C}^{0}(S)$ be the set of isotopy classes of essential simple closed curves in $S$. Let $\mathcal{V} \subset \mathcal{C}^{0}(S)$ be the set of curves which bound essential disks in the handlebody $V$. Fix also a pseudo-Anosov map $h: S \rightarrow S$.

Definition 2.2 Given $V, S$, and $h$ as above, we say that $\mathbf{H}$ holds if the stable and unstable laminations of $h, \mathcal{L}^{ \pm}(h)$, are not contained in the closure of $\mathcal{V}$ (considered as a subset of $\mathcal{P} \mathcal{M L}(S)$ ).

Definition 2.3 Given $V, S$, and $h$ as above, we say that Unb holds if the set of distances of $\left\{\left(S, \mathcal{V}, h^{n}(\mathcal{V})\right) \mid n \in \mathbb{N}\right\}$ is unbounded.

The following theorem of Hempel's 8 ] provided the first proof that high distance splittings exist.

Theorem 2.4 (Hempel) Suppose a handlebody $V$ with $S=\partial V$ and a pseudo-Anosov map $h: S \rightarrow S$ are given. Then $\mathbf{H}$ implies Unb.

Remark 2.5 Hempel cites Kobayashi [1] as the framer of the proof sketched below. However, Kobayashi used a slightly different hypothesis; see Section 11

Sketch of proof of Theorem 2.4 Suppose the distance of $\left(S, \mathcal{V}, h^{j}(\mathcal{V})\right)$ is bounded by some $M \in \mathbb{N}$, for all $j \in \mathbb{N}$. Then, for every $j$, there is a set of essential curves $\left\{\alpha_{i}^{j}\right\}_{i=0}^{M} \subset \mathcal{C}^{0}(S)$ such that

- $\alpha_{0}^{j} \in \mathcal{V}$ and $\alpha_{M}^{j} \in h^{j}(\mathcal{V})$ and

- $\alpha_{i}^{j} \cap \alpha_{i+1}^{j}=\emptyset$ for $i=0,1, \ldots, M-1$.

Thus there is a curve $\beta^{j} \in \mathcal{V}$ such that $h^{j}\left(\beta^{j}\right)=\alpha_{M}^{j}$. By $\mathbf{H}$ the unstable lamination $\mathcal{L}^{-}(h)$ is not contained in the closure of $\mathcal{V}$ (taken in $\mathcal{P} \mathcal{M L}(S)$ ). It follows that the $\beta^{j}$ 's avoid an open neighborhood of $\mathcal{L}^{-}(h)$. Thus, the curves $h^{j}\left(\beta^{j}\right)=\alpha_{M}^{j}$ converge to $\mathcal{L}^{+}(h)$ as a sequence in $\mathcal{P} \mathcal{M L}(S)$. 
Recall that $\mathcal{P} \mathcal{M L}(S)$ is compact. Inductively pass to subsequences exactly $M$ times to ensure that the $i^{\text {th }}$ sequence $\left\{\alpha_{i}^{j}\right\}_{j \in \mathbb{N}}$ also converges in $\mathcal{P} \mathcal{M L}(S)$, for $i=M-1, M-2, \ldots, 0$. Denote the limit of $\left\{\alpha_{i}^{j}\right\}_{j \in \mathbb{N}}$ by $\mathcal{L}_{i}$. Thus $\mathcal{L}_{M}=\mathcal{L}^{+}(h)$ while $\mathcal{L}_{0}$ lies in the closure of $\mathcal{V}$. In particular $\mathcal{L}_{M} \neq \mathcal{L}_{0}$ by $\mathbf{H}$.

Recall that the geometric intersection number, $\iota(\cdot, \cdot)$, extends to a continuous function $\mathcal{M L}(S) \times \mathcal{M L}(S) \rightarrow \mathbb{R}_{+}$. As $\alpha_{i}^{j} \cap \alpha_{i+1}^{j}=\emptyset$ we have $\iota\left(\mathcal{L}_{i}, \mathcal{L}_{i+1}\right)=0$, abusing notation slightly. As $\mathcal{L}^{+}(h)$ is minimal (the lamination contains no closed leaf and all complementary regions are disks) and uniquely ergodic (all transverse measures are projectively equivalent), $\mathcal{L}_{M}$ and $\mathcal{L}_{M-1}$ must be the same point of $\mathcal{P} \mathcal{M L}(S)$. Inductively, $\mathcal{L}_{i}=\mathcal{L}_{i-1}$ in $\mathcal{P} \mathcal{M L}(S)$ which implies that $\mathcal{L}_{M}=\mathcal{L}_{0}$. This is a contradiction.

\subsection{Distance grows linearly}

The primary goal of this paper, then, is to show that Hempel's hypothesis $\mathbf{H}$ implies a seemingly stronger assertion: the distance grows linearly with the number of iterates of $h$, up to a bounded additive constant. This is the condition Lin. As indicated in the introduction we do this by studying the action of $h$ on the complex of curves, $\mathcal{C}(S)$.

\section{$3 \quad$ Metric spaces}

This section briefly states the facts we need about $\delta$-hyperbolic spaces and their isometries. For a deeper discussion consult Gromov [6] or Bridson and Haefliger [2].

\subsection{Basic terminology}

Let $\left(X, d_{X}\right)$ be a metric space. If $Y$ and $Z$ are subsets of $X$ define $d_{X}(Y, Z)=$ $\inf \left\{d_{X}(y, z) \mid y \in Y, z \in Z\right\}$.

An $\operatorname{arc}$ in $X$ is a continuous map $L:[a, b] \rightarrow X$ where $[a, b]$ is a closed connected subset of the real numbers, $\mathbb{R}$. The arc $L$ is geodesic if $\left|b^{\prime}-a^{\prime}\right|=d_{X}\left(L\left(a^{\prime}\right), L\left(b^{\prime}\right)\right)$ for every finite subinterval $\left[a^{\prime}, b^{\prime}\right] \subset[a, b]$. An arc $L:[a, b] \rightarrow X$ connects two points $u, v \in X$ if $[a, b]$ is a finite interval, $L(a)=u$, and $L(b)=v$. When the choice of geodesic arc connecting $u$ to $v$ does not matter (or is clear from context) we denote it by $[u, v]$. 
This paper only considers geodesic metric spaces: metric spaces in which every pair of points is connected by some geodesic arc. However, we do not assume that our spaces are proper.

A subset $U \subset X$ is quasi-convex with constant $R \geq 0$ if, for every pair of points $u, v \in U$ and for every geodesic arc $L$ connecting $u$ to $v$, the image of $L$ lies inside a closed $R$ neighborhood of the set $U$.

An $\operatorname{arc} L:[a, b] \rightarrow X$ is quasi-geodesic with constants $\lambda \geq 1, \epsilon \geq 0$ if

$$
\frac{1}{\lambda}\left|b^{\prime}-a^{\prime}\right|-\epsilon \leq d_{X}\left(L\left(a^{\prime}\right), L\left(b^{\prime}\right)\right) \leq \lambda\left|b^{\prime}-a^{\prime}\right|+\epsilon
$$

for every finite interval $\left[a^{\prime}, b^{\prime}\right] \subset[a, b]$.

\subsection{Isometries}

A map $h: X \rightarrow X$ is an isometry if for every pair of points $x, y \in X$ we have $d_{X}(x, y)=d_{X}(h(x), h(y))$.

Definition 3.1 Fix $x \in X$. The average displacement of an isometry $h$ is the quantity

$$
\alpha(h)=\lim _{n \rightarrow \infty} \frac{d_{X}\left(x, h^{n}(x)\right)}{n}=\inf _{n \in \mathbb{N}} \frac{d_{X}\left(x, h^{n}(x)\right)}{n} .
$$

It is well-known (see 4, Chapter 10, for example) that $\alpha(h)$ exists and is independent of the given choice of $x \in X$. Note also that $n \cdot \alpha(h) \leq d_{X}\left(x, h^{n}(x)\right)$, for all $n$. We say an isometry is hyperbolic if its average displacement is strictly positive.

Remark 3.2 This is one of several equivalent definitions of a hyperbolic isometry. Claim 5.4 below shows that any orbit of a hyperbolic isometry, acting on a Gromov hyperbolic space, is a quasi-isometric embeddings of $\mathbb{Z}$, as expected.

\subsection{Gromov hyperbolicity}

A geodesic metric space $\left(X, d_{X}\right)$ is Gromov hyperbolic with constant $\delta$, or simply $\delta$-hyperbolic, if every geodesic triangle is $\delta$-thin: the (closed) $\delta$ neighborhood of any two of the sides of the triangle contains the third side. Here a geodesic triangle is a collection of three geodesic arcs which connect in pairs some triple of points $x, y, z \in X$. As an immediate corollary geodesic $n$-gons 
are $(n-2) \cdot \delta$-thin: any one side lies in a $(n-2) \cdot \delta$ neighborhood of the union of the other $n-1$ sides.

Again and again we will use the remarkable "stability" property of quasigeodesics in a $\delta$-hyperbolic space:

Lemma 3.3 For any choice of $\delta, \lambda, \epsilon$ there is a constant $R>0$ such that: if $X$ is a $\delta$-hyperbolic space $X$ and $L:[a, b] \rightarrow X$ is a $(\lambda, \epsilon)$ quasi-geodesic, then the image of $L$ is quasi-convex with constant $R$. Furthermore, if $[a, b]$ is a finite interval then the image of $L$ lies within the closed $R$ neighborhood of any geodesic connecting $L(a)$ and $L(b)$.

See 2], page 404, for a proof and note that $X$ need not be proper. The number $R$ is referred to as the stability constant for $L$.

\section{Triangles and quadrilaterals}

Following [2, page 463, we define a notion of a "quasi-projection" onto a quasiconvex set and deduce a few consequences.

\subsection{Closest point projections}

Suppose that $U \subset X$ is nonempty and $X$ is $\delta$-hyperbolic. Define a quasiprojection from $X$ to $U$ as follows: given $\epsilon>0$ and $y \in X$ put

$$
\operatorname{proj}_{U}^{\epsilon}(y)=\left\{y^{\prime} \in U \mid d_{X}\left(y, y^{\prime}\right) \leq d_{X}(y, U)+\epsilon\right\} .
$$

That is, $\operatorname{proj}_{U}^{\epsilon}(y)$ is the set of points in $U$ which are, within an error of $\epsilon$, closest to $y$. Note that $\operatorname{proj}_{U}^{\epsilon}(y)$ is nonempty.

Remark 4.1 When $U$ is quasi-convex the function $\operatorname{proj}_{U}^{\epsilon}$ is a quasi-map from $X$ to $U$. That is, the diameter of $\operatorname{proj}_{U}^{\epsilon}(y)$ is bounded independently of the point $y$. This is a direct consequence of Lemma 4.2, below.

\subsection{The geometry of projections}

This section discusses similarities between the function $\operatorname{proj}_{U}^{\epsilon}$ and orthogonal projection in hyperbolic space $\mathbb{H}^{n}$. 
Lemma 4.2 (Triangle lemma) Fix $\epsilon>0$. Suppose $X$ is $\delta$-hyperbolic and $U \subset X$ is quasi-convex with constant $R$. Suppose that $y \in X$, that $y^{\prime} \in$ $\operatorname{proj}_{U}^{\epsilon}(y)$, and that $u$ is another point of $U$. Then

$$
d_{X}\left(y, y^{\prime}\right)+d_{X}\left(y^{\prime}, u\right) \leq d_{X}(y, u)+(2 \epsilon+4 \delta+2 R) .
$$

Proof Note that if $d_{X}\left(y^{\prime}, u\right) \leq \epsilon+2 \delta+R$ then the conclusion follows from the triangle inequality. Suppose, then, that $\epsilon+2 \delta+R<d_{X}\left(y^{\prime}, u\right)$ and let $a$ be the point of $\left[y^{\prime}, u\right]$ such that $d_{X}\left(y^{\prime}, a\right)=\epsilon+2 \delta+R+\epsilon^{\prime}$, where $0<\epsilon^{\prime}<$ $d_{X}\left(y^{\prime}, u\right)-\epsilon-2 \delta-R$.

Claim 4.3 The point $a$ does not lie within a $\delta$ neighborhood of $\left[y^{\prime}, y\right]$.

Proof of Claim 4.3 Suppose the opposite. Then there is a point $b \in\left[y^{\prime}, y\right]$ with $d_{X}(a, b) \leq \delta$. See Figure 11 By the triangle inequality we have $\epsilon+\delta+R+$ $\epsilon^{\prime} \leq d_{X}\left(y^{\prime}, b\right)$. On the other hand consider a piecewise geodesic from $y$ to $b$ to $a$ to $U$. This has length at most $d_{X}(y, b)+\delta+R$ and at least $d_{X}\left(y^{\prime}, y\right)-\epsilon$. Thus $d_{X}\left(y^{\prime}, y\right)-\epsilon \leq d_{X}(y, b)+\delta+R$.

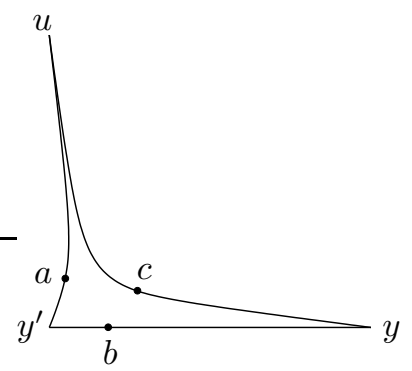

Figure 1: A "right-angled" triangle

Remark 4.4 This "no short-cuts" principle underlies both arguments in this section.

Subtract $d_{X}(y, b)$ from both sides of the above inequality to find that $d_{X}\left(y^{\prime}, b\right)$ $\leq \epsilon+\delta+R$. This contradiction proves the claim.

Continuing with the proof of Lemma 4.2, by $\delta$-thinness of the triangle $u y^{\prime} y$ we have a point $c \in[y, u]$ with $d_{X}(a, c) \leq \delta$. See Figure1 Attempting to shortcut from $y$ to $c$ to $a$ to $U$ shows that $d_{X}\left(y, y^{\prime}\right) \leq d_{X}(y, c)+\epsilon+\delta+R$. Also, by the triangle inequality applied to $u y^{\prime} c$, we have $d_{X}\left(y^{\prime}, u\right) \leq d_{X}(u, c)+\delta+\epsilon+2 \delta+$ $R+\epsilon^{\prime}$. Adding these last two inequalities and letting $\epsilon^{\prime}$ tend to zero proves the lemma. 
A similar lemma holds for quadrilaterals.

Lemma 4.5 (Quadrilateral lemma) Fix $\epsilon>0$. Suppose $X$ is a $\delta$-hyperbolic space and that $U \subset X$ is quasi-convex with constant $R$. Suppose that $y, z \in X$ while $y^{\prime} \in \operatorname{proj}_{U}^{\epsilon}(y)$ and $z^{\prime} \in \operatorname{proj}_{U}^{\epsilon}(z)$. Suppose that $2 \epsilon+8 \delta+2 R<d_{X}\left(y^{\prime}, z^{\prime}\right)$. Then

$$
d_{X}\left(y, y^{\prime}\right)+d_{X}\left(y^{\prime}, z^{\prime}\right)+d_{X}\left(z^{\prime}, z\right) \leq d_{X}(y, z)+(4 \epsilon+12 \delta+4 R) .
$$

See also Chapter 10, Proposition 2.1, of [4], Proposition III.Г.3.11 of [2, or Lemma 7.3.D of [6].

Proof of Lemma 4.5 Let $a \in\left[y^{\prime}, z^{\prime}\right]$ be the point with $d_{X}\left(a, z^{\prime}\right)=\epsilon+4 \delta+$ $R+\epsilon^{\prime}$, where $0<\epsilon^{\prime}<d_{X}\left(y^{\prime}, z^{\prime}\right)-2 \epsilon-8 \delta-2 R$. As in Claim 4.3 the point $a$ lies outside of a $2 \delta$ neighborhood of the union of $\left[y, y^{\prime}\right]$ and $\left[z, z^{\prime}\right]$. Thus, by $2 \delta$-thinness of the geodesic quadrilateral $y y^{\prime} z^{\prime} z$, there is a point $c \in[y, z]$ such that $d_{X}(a, c) \leq 2 \delta$. See Figure 2 .

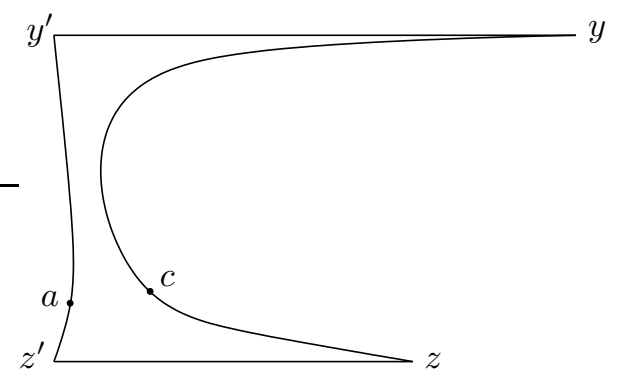

Figure 2: Quadrilateral with two "right angles"

Applying the triangle inequality to the piecewise geodesic from $y$ to $c$ to $a$ to $z^{\prime}$ we find that $d_{X}\left(y, z^{\prime}\right) \leq d_{X}(y, c)+2 \delta+\epsilon+4 \delta+R+\epsilon^{\prime}$. Combining this with Lemma 4.2 (taking $\left.u=z^{\prime}\right)$ gives $d_{X}\left(y, y^{\prime}\right)+d_{X}\left(y^{\prime}, z^{\prime}\right) \leq d_{X}(y, c)+3 \epsilon+$ $10 \delta+3 R+\epsilon^{\prime}$. Finally, short-cutting from $z$ to $c$ to $a$ to $U$ gives $d_{X}\left(z, z^{\prime}\right) \leq$ $d_{X}(z, c)+\epsilon+2 \delta+R$. Adding these last two inequalities and letting $\epsilon^{\prime}$ tend to zero gives the desired conclusion.

\section{The displacement lemma}

This section gives a proof of: 
Lemma 5.1 (Displacement lemma) Suppose $h$ is a hyperbolic isometry of the $\delta$-hyperbolic space $X$. Suppose that $x \in X$ is fixed. Then there is a constant $K$ such that, for all $n$,

$$
\left|d_{X}\left(x, h^{n}(x)\right)-n \cdot \alpha(h)\right| \leq K .
$$

Recall that $\alpha(h)$ is the average displacement of $h$; see Definition 3.1. Though this result seems to be well-known, we have not been able to find it in the literature.

Remark 5.2 Lemma 5.1 is immediate in $\mathbb{H}^{n}$. In fact $\delta$-hyperbolicity is not necessary; the displacement lemma also holds for semi-simple isometries of convex metric spaces. See [2] for definitions.

Remark 5.3 Set $\alpha_{n}=d_{X}\left(x, h^{n}(x)\right) / n$. A consequence of the lemma is that $\alpha_{n}-\alpha(h)=O(1 / n)$.

Throughout the proof of the lemma we adopt the notation $x_{n}=h^{n}(x)$ where $x_{0}=x$ is the basepoint provided by the hypothesis. Note that $n \cdot \alpha(h)-$ $d_{X}\left(x_{0}, x_{n}\right) \leq 0$. This gives the upper bound. For the lower, we define a sequence of infinite arcs in $X$ : for each positive integer $n$ fix a geodesic arc

$$
P_{n}=\left[x_{0}, x_{n}\right] .
$$

Let

$$
L_{n}=\bigcup_{k \in \mathbb{Z}} h^{n k}\left(P_{n}\right)
$$

be parameterized by arc-length. We will investigate these infinite arcs in order to prove the lemma.

Again, take $\alpha_{m}=d_{X}\left(x_{0}, x_{m}\right) / m$. Fix $M_{0} \geq 3$ so that if $m+1 \geq M_{0}$ then $\left|\alpha_{m}-\alpha(h)\right|<\frac{1}{2} \alpha(h)$. (See Definition [3.1)

Claim 5.4 The arc $L_{1}$ is a quasi-geodesic with constants $\lambda_{1}=\frac{2 \alpha_{1}}{\alpha(h)}$ and $\epsilon_{1}=M_{0} \alpha_{1}$.

See also 4, Chapter 10, Proposition 6.3.

Remark 5.5 More is true. All of the $\operatorname{arcs} L_{n}$ are quasi-geodesic with uniformly bounded additive constants $\epsilon_{n}$ and multiplicative constants of the form $\lambda_{n}=1+O(1 / n)$. 
Proof of Claim 5.4 Fix $a<b \in \mathbb{R}$. We will show that $d_{X}\left(L_{1}(a), L_{1}(b)\right)$ satisfies the inequalities given by Equation 1. To simplify notation set $L_{1}(a)=u$ and $L_{1}(b)=v$. As $L_{1}$ is parameterized by arc-length, $d_{X}(u, v) \leq|b-a|$.

By applying $h$ some number of times we may assume that $u$ lies in $P_{1}$ while $v$ lies in $h^{m}\left(P_{1}\right)$, for some smallest possible nonnegative integer $m$. Recall that $x_{m}=h^{m}(x)$. The triangle inequality gives

$$
d_{X}\left(x_{0}, x_{m}\right) \leq d_{X}(u, v)+2 \alpha_{1}
$$

where $\alpha_{1}=d_{X}\left(x_{0}, x_{1}\right)$. In the case $m+1 \geq M_{0}$ we have

$$
\frac{m}{2} \alpha(h)<d_{X}\left(x_{0}, x_{m}\right)
$$

and, as $L_{1}$ is parameterized via arc-length,

$$
|b-a| \leq(m+1) \alpha_{1} .
$$

Chaining together the above three inequalities gives:

$$
\frac{\alpha(h)}{2 \alpha_{1}}|b-a|-\frac{\alpha(h)}{2}-2 \alpha_{1} \leq d_{X}(u, v) .
$$

On the other hand, if $m+1 \leq M_{0}$ then

$$
|b-a|-M_{0} \alpha_{1} \leq d_{X}(u, v) .
$$

Since $\alpha(h) \leq \alpha_{1}$ and $M_{0} \geq 3$, regardless of $m$ we have

$$
\frac{\alpha(h)}{2 \alpha_{1}}|b-a|-M_{0} \alpha_{1} \leq d_{X}(u, v) .
$$

This completes the proof of the claim.

Definition 5.6 We call $L_{1}$ as defined in Equation 2 an invariant axis for $h$.

Returning to the proof of Lemma 5.1 choose $n \in \mathbb{N}$ and fix attention on the infinite arc $L_{n}$. Recall that $x_{m}=h^{m}(x)$ where $x$ is the chosen basepoint. As $\alpha_{m}=d_{X}\left(x_{0}, x_{m}\right) / m$ converges to $\alpha(h)$ from above there is a positive integer $M_{1}$ such that if $m>M_{1}$ then $\alpha_{m}-\alpha(h) \leq 1 / n$. Choose $m>M_{1}$ of the form $m=k n$. It follows that

$$
\frac{d_{X}\left(x_{0}, x_{m}\right)}{k}-n \cdot \alpha(h) \leq 1
$$

Now we compare the quantities $d_{X}\left(x_{0}, x_{m}\right) / k$ and $d_{X}\left(x_{0}, x_{n}\right)$. Recall that $P_{m}=\left[x_{0}, x_{m}\right]$. For $i \in\{0,1, \ldots k\}$ choose a point $z_{i} \in \operatorname{proj}_{P_{m}}^{0}\left(x_{n i}\right)$. Note that $z_{i}$ exists as $P_{m}$ is compact. It follows that $d_{X}\left(x_{n i}, z_{i}\right) \leq R$ where $R$ is the stability constant provided by Lemma 3.3 for the quasi-geodesic $L_{1}$. See Figure 3 


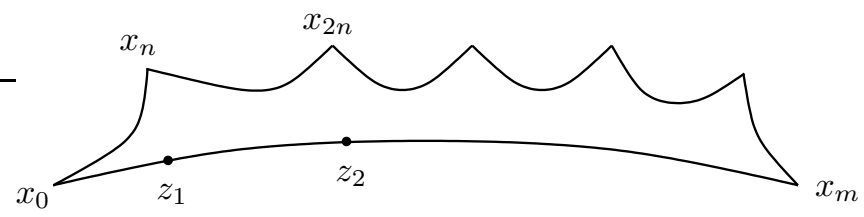

Figure 3: The sides of the ladder: recall that $x_{k}=h^{k}\left(x_{0}\right)$. The rungs connecting $x_{n i}$ to $z_{i}$ are not drawn.

Claim 5.7 If $n>\frac{3 R}{\alpha(h)}$ then, when traveling from $z_{0}=x_{0}$ to $z_{k}=x_{m}=h^{m}(x)$ along $P_{m}$, the points $z_{i}$ are distinct and encountered in order of their indices.

Proof of Claim 5.7 From the definition of $\alpha(h)$ we know that $d_{X}\left(x_{0}, x_{n j}\right) \geq$ $n \cdot \alpha(h)>3 j R$. Recall that $d_{X}\left(z_{i}, x_{n i}\right) \leq R$. So for all $i, d_{X}\left(z_{i}, z_{i+j}\right) \geq$ $3 j R-2 R$. Thus consecutive $z_{i}$ 's are distinct.

If the $z_{i}$ 's are not encountered in order by index then there is a smallest $i$ with $z_{i+1}$ appearing before $z_{i}$. So either $z_{i+1} \in\left[z_{i-1}, z_{i}\right]$ or $z_{i-1} \in\left[z_{i+1}, z_{i}\right]$. Suppose that former occurs (the latter is similar). Now, by the above paragraph, $d_{X}\left(z_{i-1}, z_{i}\right) \leq d_{X}\left(x_{0}, x_{n}\right)+2 R$ and $d_{X}\left(z_{i+1}, z_{i}\right) \geq d_{X}\left(x_{0}, x_{n}\right)-2 R$. We deduce that $d_{X}\left(z_{i-1}, z_{i+1}\right) \leq 4 R$. Thus by the triangle inequality we have $d_{X}\left(x_{n(i-1)}, x_{n(i+1)}\right) \leq 6 R$. But this is a contradiction.

To conclude the proof of Lemma 5.1] assume for the moment that $n>\frac{3 R}{\alpha(h)}$. By the above claim the intervals $\left[z_{i}, z_{i+1}\right]$ form a disjoint partition of $P_{m}$ (ignoring endpoints). Let $j \in\{0,1, \ldots, k-1\}$ be chosen to minimize the length of $\left[z_{j}, z_{j+1}\right]$. Then certainly $d_{X}\left(z_{j}, z_{j+1}\right) \leq d_{X}\left(x_{0}, x_{m}\right) / k$. Applying the triangle inequality to the rectangle with vertices $x_{n j}, z_{j}, z_{j+1}, x_{n(j+1)}$ shows that

$$
d_{X}\left(x_{0}, x_{n}\right) \leq\left(d_{X}\left(x_{0}, x_{m}\right) / k\right)+2 R .
$$

Conclude, by Equation 3, that

$$
d_{X}\left(x_{0}, x_{n}\right)-n \cdot \alpha(h) \leq 1+2 R
$$

as long as $n>\frac{3 R}{\alpha(h)}$. Thus regardless of $n$

$$
\left|d_{X}\left(x_{0}, x_{n}\right)-n \cdot \alpha(h)\right| \leq \max \left\{1+2 R, \frac{3 R \alpha_{1}}{\alpha(h)}\right\} .
$$

This proves the Displacement Lemma. 


\section{Bounded projection implies linear growth}

Let $X$ be a $\delta$-hyperbolic space and $h$ a hyperbolic isometry of $X$. Choose an invariant axis $L_{1}$ for $h$ as in Definition 5.6 above.

Definition 6.1 A subset $Y \subset X$ has bounded projection with respect to $h$ if there is $\epsilon>0$ so that the set $P=\bigcup_{z \in Y} \operatorname{proj}_{L_{1}}^{\epsilon}(z)$ has finite diameter.

Note that (for given $Y$ and $h$ ) the above definition is independent of the choices involved in defining $L_{1}$. In this section we prove:

Theorem 6.2 If $Y$ has bounded projection with respect to $h$ then there is a constant $K$ such that:

$$
\left|d_{X}\left(Y, h^{n}(Y)\right)-n \cdot \alpha(h)\right| \leq K .
$$

Proof Pick any point $y \in Y$. Then there is a constant $K^{\prime}$, provided by Lemma 5.1] such that $d_{X}\left(y, h^{n}(y)\right)-n \cdot \alpha(h) \leq K^{\prime}$ for all $n$. So $d_{X}\left(Y, h^{n}(Y)\right)-$ $n \cdot \alpha(h) \leq K^{\prime}$ for all $n$. This gives the desired upper bound. We now turn to the lower bound.

Let $y \in Y$ and take $z \in h^{n}(Y)$. We must bound from below the quantity $d_{X}(y, z)$. Choose $y^{\prime} \in \operatorname{proj}_{L_{1}}^{\epsilon}(y)$ and $z^{\prime} \in \operatorname{proj}_{L_{1}}^{\epsilon}(z)$. Let $P=\bigcup_{w \in Y} \operatorname{proj}_{L_{1}}^{\epsilon}(w)$ be the projection of $Y$ to $L_{1}$. By hypothesis this set has finite diameter, say $K^{\prime \prime}$. Note that $d_{X}\left(z^{\prime}, h^{n}\left(y^{\prime}\right)\right) \leq K^{\prime \prime}$.

By Definition 3.1 we have $n \cdot \alpha(h) \leq d_{X}\left(y^{\prime}, h^{n}\left(y^{\prime}\right)\right)$. Thus, by the triangle inequality, $n \cdot \alpha(h) \leq d_{X}\left(y^{\prime}, z^{\prime}\right)+K^{\prime \prime}$.

For the remainder of the proof take $M \equiv \frac{2 \epsilon+8 \delta+2 R+K^{\prime \prime}}{\alpha(h)}$. Then, when $n>M$ the previous inequality implies that $2 \epsilon+8 \delta+2 R<d_{X}\left(y^{\prime}, z^{\prime}\right)$. Letting $K^{\prime \prime \prime}=$ $4 \epsilon+12 \delta+4 R$ and applying Lemma 4.5 we have

$$
\begin{aligned}
d_{X}(y, z)+K^{\prime \prime \prime} & \geq d_{X}\left(y, y^{\prime}\right)+d_{X}\left(y^{\prime}, z^{\prime}\right)+d_{X}\left(z^{\prime}, z\right) \\
& \geq d_{X}\left(y^{\prime}, z^{\prime}\right) .
\end{aligned}
$$

Thus when $n>M$, regardless of our choice of $y \in Y$ and $z \in h^{n}(Y)$, we have

$$
n \cdot \alpha(h) \leq d_{X}(y, z)+K^{\prime \prime}+K^{\prime \prime \prime} .
$$

That is,

$$
n \cdot \alpha(h)-d_{X}\left(Y, h^{n}(Y)\right) \leq K^{\prime \prime}+K^{\prime \prime \prime}
$$


for $n>M$. Also, as discussed in the first paragraph of the proof,

$$
d_{X}\left(Y, h^{n}(Y)\right)-n \cdot \alpha(h) \leq K^{\prime}
$$

for all $n$. So take

$$
K=\max \left\{K^{\prime}, K^{\prime \prime}+K^{\prime \prime \prime},\left|d_{X}\left(Y, h^{n}(Y)\right)-n \cdot \alpha(h)\right|\right\}
$$

where $n$ ranges from 1 to $M$. This gives the desired bound.

\section{$7 \quad$ B implies Lin}

This section transforms the preceding purely geometric considerations into tools appropriate to the setting of Heegaard splittings. We begin by defining the graph of curves.

Let $S$ be a closed orientable surface of genus at least two. Let $\mathcal{C}^{0}(S)$ be the set of isotopy classes of essential simple closed curves in $S$. The graph of curves, $\mathcal{C}^{1}(S)$, has vertex set $\mathcal{C}^{0}(S)$ and an edge connecting two distinct vertices if and only if the two curves may be realized disjointly. We take each such edge to be a copy of the interval $[0,1]$ and give $\mathcal{C}^{1}(S)$ the induced metric.

Remark 7.1 The graph $\mathcal{C}^{1}(S)$ is the one-skeleton of the curve complex and is quasi-isometric to the full complex. For simplicity we consider only $\mathcal{C}^{1}(S)$.

We require a pair of deep results of Masur and Minsky [14].

Theorem 7.2 (Masur-Minsky) The graph of curves $\left(\mathcal{C}^{1}(S), d_{\mathcal{C}}\right)$, is a Gromov hyperbolic space. Furthermore, pseudo-Anosov maps act on the graph of curves as hyperbolic isometries.

Thus, Theorem 6.2 can be translated to the language of Heegaard splittings as follows. Recall that $\mathcal{V} \subset \mathcal{C}^{0}(S)$ is the set of curves which bound essential disks in the handlebody $V$. As usual $S=\partial V$ has genus two or more. Fix $h: S \rightarrow S$ a pseudo-Anosov map.

Definition 7.3 Given $V, S$, and $h$ as above, we say that $\mathbf{B}$ holds if the handlebody set $\mathcal{V} \subset \mathcal{C}^{1}(S)$ has bounded projection with respect to $h$.

Also, note that the distance of a Heegaard splitting $(S, \mathcal{V}, \mathcal{W})$ (Definition 2.1) is exactly the quantity $d_{\mathcal{C}}(\mathcal{V}, \mathcal{W})$. 
Definition 7.4 Given $V, S$, and $h$ as above, we say that Lin holds if there is a constant $K$ so that, for all $n \in \mathbb{N}$,

$$
\left|d_{\mathcal{C}}\left(\mathcal{V}, h^{n}(\mathcal{V})\right)-n \cdot \alpha(h)\right| \leq K .
$$

Corollary 7.5 Suppose a handlebody $V$ with $S=\partial V$ and a pseudo-Anosov map $h: S \rightarrow S$ are given. Then B implies Lin.

Proof This follows immediately from Theorems 6.2 and 7.2

Remark 7.6 Algorithmic computation of $d_{\mathcal{C}}\left(\mathcal{V}, h^{n}(\mathcal{V})\right)$ would be highly desirable. Upper and lower bounds for $\alpha(h)$ may perhaps be obtained using methods similar to [14]. Estimation of $K$ seems more difficult. For any $n \in \mathbb{N}$ there are pairs $(V, h)$ where the projection of $\mathcal{V}$ to $L_{1}$ has finite diameter which is bigger than $n$.

\section{Equivalence of $\mathrm{H}$ and $\mathrm{B}$}

In this section we deduce the following from work of Klarreich [10].

Theorem 8.1 Suppose a handlebody $V$ with $S=\partial V$ and a pseudo-Anosov map $h: S \rightarrow S$ are given. Then $\mathbf{H}$ is equivalent to $\mathbf{B}$.

\subsection{The Gromov product}

Before proving Theorem 8.1 we recall the definition of the Gromov product. Suppose that a basepoint $x_{0}$ in the $\delta$-hyperbolic space $X$ is given. The Gromov product of a pair of points $y, z \in X$ is the quantity

$$
(y \cdot z)=\frac{1}{2}\left(d_{X}\left(x_{0}, y\right)+d_{X}\left(x_{0}, z\right)-d_{X}(y, z)\right) .
$$

Following [6] we say that a sequence $\left\{y_{i}\right\}_{i=0}^{\infty} \subset X$ converges at infinity if $\lim _{n, m \rightarrow \infty}\left(y_{n} \cdot y_{m}\right)$ is infinite. This is independent of the choice of basepoint, $x_{0}$. Two such sequences $\left\{y_{i}\right\}$ and $\left\{z_{i}\right\}$, both converging at infinity, are equivalent if $\lim _{n, m \rightarrow \infty}\left(y_{n} \cdot z_{m}\right)$ is again infinite. The Gromov boundary of $X$, denoted $\partial_{\infty} X$, is the set of equivalence classes of sequences which converge at infinity. As a final bit of notation, set $|y|=(y \cdot y)=d_{X}\left(x_{0}, y\right)$.

\footnotetext{
${ }^{1}$ Added in proof: K Shackleton [16] using work of B Bowditch [1] has made significant progress on this problem.
} 
Now, if $h$ is a hyperbolic isometry of $X$ we define the stable and unstable fixed points of $h$ to be the points of $\partial_{\infty} X$ containing the sequences $L^{+}(h)=\left\{x_{n}=\right.$ $\left.h^{n}\left(x_{0}\right) \mid n \in \mathbb{N}\right\}$ and $L^{-}(h)=\left\{x_{-n}=h^{-n}\left(x_{0}\right) \mid n \in \mathbb{N}\right\}$ respectively. Recall that $L_{1}$ is a piecewise geodesic through the points $\left\{x_{i}\right\}_{i=-\infty}^{\infty}$.

There is a simple relation between projection to the quasi-geodesic $L_{1}$ and the Gromov product. Fix $\epsilon>d_{X}\left(x_{0}, x_{1}\right)$. Again we use $x_{0}$ as the basepoint for computing the Gromov product.

Lemma 8.2 Fix $y, z \in X$. Pick $x_{m} \in \operatorname{proj}_{L_{1}}^{\epsilon}(y), x_{n} \in \operatorname{proj}_{L_{1}}^{\epsilon}(z)$, and suppose that $n, m>0$. Then

$$
(y \cdot z) \geq \min \left\{\left|x_{n}\right|,\left|x_{m}\right|\right\}-K
$$

and

$$
\left|\left(y \cdot x_{n}\right)-\min \left\{\left|x_{n}\right|,\left|x_{m}\right|\right\}\right| \leq K .
$$

Here $K$ is a constant not depending on $y$ or $z$.

Proof Suppose that $m \leq n$ as the other case is similar. For ease of notation let $A=d_{X}\left(x_{0}, x_{m}\right)=\left|x_{m}\right|, B=d_{X}\left(x_{m}, y\right), C=d_{X}\left(x_{m}, x_{n}\right), D=d_{X}\left(x_{n}, z\right)$, and $E=d_{X}\left(x_{0}, x_{n}\right)=\left|x_{n}\right|$. Let $K^{\prime}=2 \epsilon+4 \delta+2 R$, where $R$ is the stability constant for $L_{1}$.

Now $(y \cdot z)=\frac{1}{2}\left(d_{X}\left(x_{0}, y\right)+d_{X}\left(x_{0}, z\right)-d_{X}(y, z)\right)$. The first term is greater than $A+B-K^{\prime}$, applying Lemma 4.2 and the fact that $L_{1}$ is quasi-geodesic (Claim 5.4), hence quasi-convex. Similarly, the second term is greater than $D+E-K^{\prime}$. But $E \geq C+A-2 R$, using the triangle inequality and the fact that $L_{1}$ is a quasi-geodesic. Finally, the third term is less than $B+C+D$ using the triangle inequality. So $(y \cdot z) \geq \frac{1}{2}\left(A+B-K^{\prime}+A+C+D-2 R-K^{\prime}-B-C-D\right)$ and we have the desired lower bound.

When $z=x_{n}$ we also obtain an upper bound, as in this case the first term is less than $A+B$, the second is less than $A+C$, while the third is greater than $B+C-K^{\prime}$.

This leads to:

Lemma 8.3 A subset $Y \subset X$ has unbounded projection with respect to $h$ if and only if there is a sequence $y_{n} \in Y$ converging to the stable or unstable fixed point for $h$ at infinity. 
Proof Suppose that the set $Y$ has unbounded projection to the sequence $L^{+}(h)=\left\{x_{n}=h^{n}\left(x_{0}\right) \mid n \in \mathbb{N}\right\}$. (The other case is similar.) Choose $\epsilon>$ $d_{X}\left(x_{0}, x_{1}\right)$ and a sequence $\left\{y_{n}\right\} \subset Y$ so that $x_{m(n)} \in \operatorname{proj}_{L_{1}}^{\epsilon}\left(y_{n}\right)$, for some $m(n)>n$. It follows from Lemma 8.2 that $\left(y_{k} \cdot y_{l}\right) \geq \min \left\{\left|x_{m(k)}\right|,\left|x_{m(l)}\right|\right\}-$ $K$ and thus $\left\{y_{n}\right\}$ converges at infinity. Also, using the second inequality of Lemma 8.2 it is easy to check that $\left\{y_{n}\right\}$ and $\left\{x_{m(n)}\right\}$ are equivalent.

On the other hand, suppose that there is a sequence $\left\{y_{n}\right\} \subset Y$ with $\left\{y_{n}\right\}$ converging to the stable fixed point of $h$. Then $\left\{y_{n}\right\}$ and $\left\{x_{n}\right\}$ are equivalent. So we may pass to subsequences $\left\{y_{k}\right\}$ and $\left\{x_{k}\right\}$ so that $\left(y_{k} \cdot x_{k}\right)$ goes to infinity with $k$. Pick $x_{m(k)} \in \operatorname{proj}_{L_{1}}^{\epsilon}\left(y_{k}\right)$. Then, by the second half of Lemma 8.2, the quantity $\min \left\{\left|x_{m(k)}\right|,\left|x_{k}\right|\right\}$ must also tend to infinity with $k$. Thus $\left|x_{m(k)}\right|$ also tends to infinity with $k$ and we are done.

\subsection{The boundary of the curve complex}

We next cite the necessary component from Klarreich [10]. Let MinLam be the space of minimal measured laminations, considered up to topological equivalence (ie, take a quotient by forgetting the measures). Klarreich gives a homeomorphism $\pi$ : MinLam $\rightarrow \partial_{\infty} \mathcal{C}^{1}(S)$ such that the following holds:

Theorem 8.4 (Klarreich) Let $\gamma_{n}$ be a sequence of essential simple closed curves in the surface $S$. Suppose that $\mathcal{L}$ is a minimal, uniquely ergodic lamination on $S$. Then the sequence $\gamma_{n} \in \mathcal{P} \mathcal{M L}(S)$ converges to $\mathcal{L}$ if and only if $\gamma_{n} \in \mathcal{C}^{1}(S)$ converges to $\pi(\mathcal{L}) \in \partial_{\infty} \mathcal{C}^{1}(S)$.

See Theorem 3.2 of [15], for a more precise version of Klarreich's result. We are now ready to prove Theorem 8.1 .

Proof of Theorem 8.1] To begin, pick any $x \in \mathcal{C}^{0}(S)$, and let $L_{1}$ be a quasigeodesic (as defined in Section 5) passing through the points $\left\{x_{n}=h^{n}(x)\right.$ $n \in \mathbb{Z}\}$. Also take $x=x_{0}$ to be the basepoint when computing the Gromov product.

Note that $\mathbf{H}$ does not hold if and only if there is a sequence of curves $v_{n} \in \mathcal{V}$ such that $v_{n}$ converges in $\mathcal{P} \mathcal{M L}(S)$ to one of $\mathcal{L}^{ \pm}(h)$. Suppose $v_{n}$ converges to $\mathcal{L}^{+}(h)$. (The other case is identical.)

\footnotetext{
${ }^{2}$ Added in proof: U Hamenstaedt [7] has announced a combinatorial proof of Klarreich's theorem.
} 
Applying Theorem 8.4 the $v_{n}$ converge in $\mathcal{P} \mathcal{M L}(S)$ to $\mathcal{L}^{+}(h)$ if and only if they also converge, in $\mathcal{C}^{1}(S)$, to $\pi\left(\mathcal{L}^{+}(h)\right)$ in the boundary of the curve complex. Now apply Lemma 8.3 to find that this occurs if and only if $\mathcal{V}$ has unbounded projection to $L^{+}(h)$.

\section{The converse: Unb implies B}

In this section we prove Theorem 9.3. Unb implies B. Recall that $\mathcal{V} \subset \mathcal{C}^{0}(S)$, the handlebody set, contains all curves which bound disks in the handlebody $V$. We will need a final result of Masur and Minsky [13]:

Theorem 9.1 (Masur-Minsky) Fix a handlebody $V$ with $\partial V=S$. The handlebody set $\mathcal{V}$ is quasi-convex in $\mathcal{C}^{1}(S)$.

We need one preparatory lemma about quasi-convex sets.

Lemma 9.2 Suppose $X$ is a $\delta$-hyperbolic space and $Y$ and $Z$ are quasiconvex subsets with constant $R$. There is a constant $K$, depending only on $\delta$ and $R$, such that: if $\left\{y_{m}\right\} \subset Y$ and $\left\{z_{n}\right\} \subset Z$ converge to the same point of $\partial_{\infty} X$ then $d_{X}(Y, Z)<K$.

To paraphrase: if $Y$ and $Z$ are quasi-convex and intersect at infinity then $Y$ and $Z$ are close to each other.

Proof Pick $y_{0}$ to be the basepoint for computing the Gromov product. Set $D=d_{X}\left(y_{0}, z_{0}\right)$. As $\lim _{m, n \rightarrow \infty}\left(y_{m} \cdot z_{n}\right)=\infty$ we also have $\lim _{m \rightarrow \infty}\left(y_{m} \cdot z_{m}\right)=\infty$. Thus there is a large $k>0$ so that any geodesic $\left[y_{k}, z_{k}\right]$ lies outside of a $5 \delta+D$ ball about $y_{0}$.

Consider, then, a geodesic quadrilateral with vertices, in order, $y_{0}, z_{0}, z_{k}$, and $y_{k}$. Recall that the quadrilateral is $2 \delta$-thin: any one side lies in the $2 \delta$ neighborhood of the union of the other three sides. Apply this to the side $\left[y_{0}, y_{k}\right]$. But the $2 \delta$ neighborhood of $\left[y_{0}, z_{0}\right]$ lies within the $2 \delta+D$ ball about $y_{0}$. Also, the $2 \delta$ neighborhood of $\left[y_{k}, z_{k}\right]$ lies without the $3 \delta+D$ ball about $y_{0}$. Thus some point of $\left[y_{0}, y_{k}\right]$ lies within $2 \delta$ of some point of $\left[z_{0}, z_{k}\right]$. Apply quasi-convexity to find that $d_{X}(Y, Z)<2 \delta+2 R$.

We may now prove: 
Theorem 9.3 Suppose a handlebody $V$ with $S=\partial V$ and a pseudo-Anosov map $h: S \rightarrow S$ are given. Then Unb implies B.

Proof We prove the contrapositive. Choose $x \in \mathcal{C}^{0}(S)$ to be the basepoint and construct $L_{1}$, a piecewise geodesic connecting the points $\left\{x_{n}=h^{n}(x)\right\}$, as in Section [5. Suppose that $\mathcal{V}$ has unbounded projection to $L_{1}$. Thus, by Lemma 8.3. there is a sequence $v_{m} \in \mathcal{V}$ converging to the stable fixed point for $h$ at infinity. (The case where $v_{m}$ converges to the unstable fixed point for $h$ is identical.)

Note that, for any fixed $n \in \mathbb{Z}$, the same holds of the handlebody set $h^{n}(\mathcal{V})$, as unbounded projection to $L_{1}$ is $h$-invariant. By Theorem 9.1 both $\mathcal{V}$ and $h^{n}(\mathcal{V})$ are quasi-convex. Thus both requirements of Lemma 9.2 are satisfied. It follows that the distance $d_{\mathcal{C}}\left(\mathcal{V}, h^{n}(\mathcal{V})\right)$ is bounded independently of $n$.

\section{Equivalence of $\mathrm{M}$ and $\mathrm{H}$}

As usual we have a handlebody $V$ of genus at least two, $S=\partial V$, and $h$ a pseudo-Anosov map on $S$. The following subset was introduced by Masur in 12 .

Definition 10.1 The Masur domain $\mathcal{M}(V) \subset \mathcal{P} \mathcal{M L}(S)$ is the set of laminations having nonzero geometric intersection with every lamination in the closure of $\mathcal{V}$.

Definition 10.2 Given $V, S$, and $h$ as above, we say that $\mathbf{M}$ holds if both the stable and unstable laminations of $h$ lie in $\mathcal{M}(V)$.

We have:

Lemma 10.3 Suppose a handlebody $V$ with $S=\partial V$ and a pseudo-Anosov map $h: S \rightarrow S$ are given. Then $\mathbf{M}$ is equivalent to $\mathbf{H}$.

Proof Any lamination has zero geometric intersection with itself. So the closure of $\mathcal{V}$ lies in the complement of $\mathcal{M}(V)$. Thus $\mathbf{M}$ implies $\mathbf{H}$.

Now consider the stable or unstable lamination of $h, \mathcal{L}^{ \pm}(h)$. As $\mathcal{L}^{ \pm}(h)$ is minimal and uniquely ergodic, $\iota\left(\mu, \mathcal{L}^{ \pm}(h)\right)=0$ implies that $\mu=\mathcal{L}^{ \pm}(h)$ as projective measured laminations. Thus if $\mathcal{L}^{ \pm}(h)$ is not in the Masur domain of $V$ then it must be in the closure of $\mathcal{V}$. 
Distances of Heegaard splittings

\section{Equivalence of $\mathrm{K}$ and $\mathrm{H}$}

In [1] Kobayashi gives several examples of pairs $(V, h)$ satisfying a condition which we call $\mathbf{K}$ (see Definition 11.4). In this section we prove that $\mathbf{K}$ is equivalent to Hempel's hypothesis $\mathbf{H}$.

\subsection{Laminations and the Whitehead graph}

A cut system $C$ for a handlebody $V$ is a collection of disjoint, nonparallel, essential disks in $V$ so that the closure of $V \backslash C$ (in the path metric) is a union of three-balls. A maximal cut system will be referred to as a pants decomposition of $V$. If $V \backslash C$ is connected then $C$ is a minimal cut system. Recall that $S=\partial V$. Let $\mathcal{L}$ be a measured lamination on $S$.

Definition 11.1 The lamination $\mathcal{L}$ is tight with respect to a cut system $C$ if no component of $S \backslash(C \cup \mathcal{L})$ is trivial: has boundary a union of two arcs, $\alpha \cup \beta$, where $\alpha \cap \beta=\partial \alpha=\partial \beta, \alpha \subset \mathcal{L}$ and $\beta \subset \partial C$.

When $C$ and $\mathcal{L}$ are tight we form the Whitehead graph $\Gamma(\mathcal{L}, C)$ as follows. Let $P$ be the closure of $S \backslash \partial C$, in the path metric. Thus $P$ is a disjoint union of planar surfaces. Every arc of $\mathcal{L} \cap P$ now falls into one of finitely many homotopy classes of properly embedded $\operatorname{arcs}$ in $P$. The vertices of $\Gamma(\mathcal{L}, C)$ are the boundary components of $P$. For every homotopy class of arc we have an edge with the obvious endpoints. Note that every such arc inherits a positive transverse measure from $\mathcal{L}$. Note that the number of components of $\Gamma(\mathcal{L}, C)$ equals the number of components of $P$, when $\mathcal{L}$ is minimal.

A component of $\partial P$ is a cut vertex for the graph if removing the component, and all (open) edges adjacent to it, increases the number of components of $\Gamma(\mathcal{L}, C)$.

Definition 11.2 A minimal lamination $\mathcal{L}$ is of full type with respect to $C$ if $\mathcal{L}$ and $C$ are tight and the associated Whitehead graph has no cut vertex.

One obstruction to being full type is the presence of waves.

Definition 11.3 A wave is any component of $\mathcal{L} \backslash C$ giving a loop-edge in the Whitehead graph $\Gamma(\mathcal{L}, C)$. 


\subsection{Kobayashi's hypothesis}

Let $V$ be a handlebody (of genus at least two) and $h$ a pseudo-Anosov map from $S=\partial V$ to itself. From [1] we have:

Definition 11.4 Given $V, S$, and $h$ as above, we say that $\mathbf{K}$ holds if there are pants decompositions $C^{ \pm}$such that $\mathcal{L}^{ \pm}(h)$ is of full type with respect to $C^{ \pm}$.

Theorem 11.5 Suppose a handlebody $V$ with $S=\partial V$ and a pseudo-Anosov map $h: S \rightarrow S$ are given. Then $\mathbf{K}$ is equivalent to $\mathbf{H}$.

We prove the two directions separately.

Lemma 11.6 If $\mathbf{K}$ holds then $\mathbf{H}$ holds.

Proof Suppose $\mathbf{K}$ holds. We fix attention on $\mathcal{L}^{+}(h)$ and the maximal cut system $C^{+}$, as the other case is identical. Let $v_{n}$ be a sequence from $\mathcal{V}$, converging in $\mathcal{P} \mathcal{M L}(S)$ to some minimal lamination, $\mathcal{L}$. We must show that $\mathcal{L} \neq \mathcal{L}^{+}(h)$.

Isotope each of the $v_{n}$ 's so that each is tight with respect to $C^{+}$. Let $P$ be the collection of pants obtained by cutting $S$ along $\partial C^{+}$and taking the closure in the path metric. Passing to a subsequence, if necessary, we may assume that every pair $\left(v_{n}, C^{+}\right)$yields the same Whitehead graph, $\Gamma=\Gamma\left(v_{n}, C^{+}\right)$. Note that $\Gamma$ contains $\Gamma^{\prime}=\Gamma\left(\mathcal{L}, C^{+}\right)$as a subgraph.

As the $v_{n}$ 's bound disks, there is some component $\rho$ of $\partial P$ so that every $v_{n}$ contains a wave for $\rho$. It follows that $\rho$ is a cut vertex for $\Gamma$ and hence for $\Gamma^{\prime}$. So $\mathcal{L}$ is not of full type and cannot be equal to $\mathcal{L}^{+}(h)$.

The converse is more difficult and is dealt with in two steps.

Lemma 11.7 If $\mathbf{H}$ holds then there are minimal cut systems $C^{ \pm}$so that $\mathcal{L}^{ \pm}(h)$ is full type with respect to $C^{ \pm}$.

Proof We prove the contrapositive. Suppose, as the other case is similar, that the stable lamination $\mathcal{L}^{+}(h)$ fails to be full type for every single minimal cut system in $V$. Fix a measure on $\mathcal{L}^{+}(h)$. Fix attention on a single minimal cut system $C$. As above let $P$ be the closure of $S \backslash \partial C$ in the path metric. Let 
$\Gamma=\Gamma\left(\mathcal{L}^{+}(h), C\right)$ be the Whitehead graph. By hypothesis there is a disk $D \in C$ giving $\Gamma$ a cut vertex.

As $D$ gives a cut vertex, there is an essential arc $\gamma$ properly embedded in $P$ with $\gamma \cap \mathcal{L}^{+}(h)=\emptyset$. We use $\gamma$ to do a disk replacement: choose a properly embedded arc $\delta \subset D$ joining the endpoints of $\gamma$. As $V \backslash C$ is a ball the curve $\gamma \cup \delta$ bounds a disk $E$. Let $D^{\prime}, D^{\prime \prime}$ be the components of $D \backslash \delta$. One of the disks $D^{\prime} \cup E$ or $D^{\prime \prime} \cup E$ is nonseparating in $V$. Thus form a new minimal cut system $C^{\prime}$ by removing $D$ and adding this new disk.

Note that $\iota\left(C^{\prime}, \mathcal{L}^{+}(h)\right)<\iota\left(C, \mathcal{L}^{+}(h)\right)$, by minimality of $\mathcal{L}^{+}(h)$. (A cut system is a measured lamination when given the counting measure.) Also, by hypothesis, $\mathcal{L}^{+}(h)$ again fails to be of full type with respect to $C^{\prime}$. So we may produce a sequence of cut systems, $C^{(n)}$, which have decreasing intersection number with $\mathcal{L}^{+}(h)$. It follows that $C^{(n)}$ is unbounded in $\mathcal{M L}(S)$.

Choose a sequence $r_{n} \in \mathbb{R}_{+}$(with $r_{n} \rightarrow 0$ ) so that the sequence of measured laminations $\left\{r_{n} \cdot C^{(n)}\right\}$ is bounded in $\mathcal{M L}(S) \backslash 0$. Passing to a convergent subsequence let $\mathcal{L}=\lim \left(r_{n} C^{(n)}\right)$. As $\iota\left(C^{(n)}, \mathcal{L}^{+}(h)\right)$ is bounded, $\iota\left(\mathcal{L}, \mathcal{L}^{+}(h)\right)=$ 0 . As in the proof of Theorem 2.4. minimality and unique ergodicity imply that $\mathcal{L}^{+}(h)=\mathcal{L}$ and $\mathbf{H}$ does not hold.

Lemma 11.8 If a minimal lamination $\mathcal{L}$ is full type with respect to some minimal cut system then $\mathcal{L}$ is full type with respect to some maximal cut system.

Proof We will prove that if $\mathcal{L}$ is full type with respect to a non-maximal cut system $C \subset V$ then there is a disk $D \subset V$ so that $C^{\prime}=C \cup\{D\}$ is again a cut system and $\mathcal{L}$ remains of full type.

Recall that $P$ is the union of planar surfaces obtained by cutting $S=\partial V$ along $C$. Let $\hat{P}$ be the quotient of $P$ obtained by identifying each boundary component to a point. Note that $\Gamma=\Gamma(\mathcal{L}, C)$ is naturally embedded in $\hat{P}$. Let $q: P \rightarrow \hat{P}$ be the quotient map.

Now, a bigon is any cycle in $\Gamma$ of length two. A cut edge is any edge such that removing the edge, its endpoints, and all edges adjacent to it, from $\Gamma$ increases the number of components of $\Gamma$. Note that if an edge lies on a bigon then it is a cut edge. For every cut edge $E$ which does not lie on a bigon we may add an extra edge $E^{\prime} \subset \hat{P}$ to $\Gamma$, with $E^{\prime} \cap \Gamma=\partial E^{\prime}$, to form a temporary bigon. Note also that these extra edges may be added disjointly, as all simple closed curves in the sphere separate. 
Let $B$ be an innermost bigon (either temporary or not) in $\hat{P}$. (If there are none set $B=\emptyset$.) Let $Q$ be the component of $\hat{P} \backslash B$ which meets no bigons. Note that none of the extra edges lie in $Q$. So choose any edge $F \subset \Gamma$ with interior contained in the interior of $Q$. Let $D^{\prime \prime}$ be a regular neighborhood of $F$, taken in $\hat{P}$. Let $D^{\prime}=q^{-1}\left(D^{\prime \prime}\right) \subset P$. Let $\delta=\partial D^{\prime} \backslash \partial P$. Let $D$ be a disk in $V$ such that $\partial D=\delta$.

Let $C^{\prime}=C \cup\{D\}$ and let $\Gamma^{\prime}=\Gamma\left(\mathcal{L}, C^{\prime}\right)$. (So $\Gamma^{\prime}$ is essentially obtained from $\Gamma$ by collapsing the edge $F$ and removing surplus parallel arcs.) Now, $\Gamma^{\prime}$ cannot have a cut vertex anywhere except at $D$; any such vertex would give a cut vertex for $\Gamma$. On the other hand, if $D$ gives a cut vertex for $\Gamma^{\prime}$ then the edge $F$ was either part of a bigon (and $\Gamma^{\prime}$ has a wave) or $F$ was part of a temporary bigon (and $\Gamma^{\prime}$ has a cut vertex without waves.) This would contradict our choice of $F$.

Now $\mathbf{H}$ implies $\mathbf{K}$ by Lemma 11.7 and Lemma 11.8. This completes the proof of Theorem 11.5, and thus of Theorem 1.1.

\section{References}

[1] Brian H Bowditch, Tight geodesics in the curve complex, http://www.maths.soton.ac.uk/staff/Bowditch/preprints.html

[2] Martin R Bridson, André Haefliger, Metric spaces of non-positive curvature, Grundlehren series 319, Springer-Verlag, Berlin (1999) MathReview

[3] Andrew J Casson, Cameron McA Gordon, Reducing Heegaard splittings, Topology Appl. 27 (1987) 275-283 MathReview

[4] M Coornaert, T Delzant, A Papadopoulos, Géométrie et théorie des groupes: Les groupes hyperboliques de Gromov, Lecture Notes series 1441, Springer-Verlag, Berlin (1990) MathReview

[5] Fathi, Laudenbach, Poénaru (editors), Travaux de Thurston sur les surfaces, Séminaire Orsay, Astérisque 66-67, Soc. Math. France, Paris (1979) MathReview

[6] Mikhael Gromov, Hyperbolic groups, from: "Essays in group theory", Math. Sci. Res. Inst. Publ 8, Springer, New York (1987) 75-263 MathReview

[7] U Hamenstaedt, Train tracks and the Gromov boundary of the complex of curves, arXiv:math.GT/0409611

[8] John Hempel, 3-manifolds as viewed from the curve complex, Topology 40 (2001) 631-657 MathReview 
[9] Michael Kapovich, Hyperbolic manifolds and discrete groups, Progress in Mathematics 183, Birkhäuser, Boston, MA (2001) MathReview

[10] Erica Klarreich, The boundary at infinity of the curve complex and the relative Teichmüller space, http://nasw.org/users/klarreich/research.htm

[11] Tsuyoshi Kobayashi, Heights of simple loops and pseudo-Anosov homeomorphisms, from: "Braids (Santa Cruz, CA, 1986)", Contemp. Math. 78, Amer. Math. Soc. Providence, RI (1988) 327-338 MathReview

[12] Howard Masur, Measured foliations and handlebodies, Ergodic Theory Dynam. Systems 6 (1986) 99-116 MathReview

[13] Howard A Masur, Yair N Minsky, Quasiconvexity in the curve complex arXiv:math.GT/0307083

[14] Howard A Masur, Yair N Minsky, Geometry of the complex of curves. I. Hyperbolicity, Invent. Math. 138 (1999) 103-149 MathReview

[15] Yair N Minsky, Combinatorial and Geometrical Aspects of Hyperbolic 3Manifolds, from: "Kleinian groups and hyperbolic 3-manifolds (Warwick, 2001)", LMS Lecture Note Ser. 299, Cambridge Univ. Press (2003) 3-40 MathReview

[16] Kenneth J Shackleton, Tightness and computing distances in the curve complex, arXiv:math.GT/0412078 